\title{
EXACT EIGENSTATES OF THE PAIRING-FORCE HAMILTONIAN
}

\author{
R. W. RICHARDSON ${ }^{\dagger}$ and N. SHERMAN
}

H. M. Randall Laboratory of Physics, University of Michigan, Ann Arbor, Michigan ${ }^{\dagger \dagger}$

\author{
Received 23 September 1963
}

\begin{abstract}
The problem of determining the eigenstates of the pairing-force Hamiltonian is reformulated in terms of the eigenstates of a many-boson system with an $N$-body interaction. The $N$-body interaction includes the effects of the Pauli principle on the eigenstates of the pairing-force Hamiltonian. Explicit expressions for four types of eigenstates are derived. These four types are the eigenstates of $N$ pairs in one or two multiply degenerate single-particle levels, the one-pair eigenstates and a new restricted class of $N$-pair eigenstates.
\end{abstract}

\section{Introduction}

The motivation for the study of the exact eigenstates of the pairing-force Hamiltonian originates in some recent models of the nucleus $\left.{ }^{1-7}\right)$. In these models, it is assumed that the nucleons in closed shells or those with energies well below the Fermi energy of the system are well described by an independent-particle model, e.g., the shell model ${ }^{8}$ ) or the Nilsson-model ${ }^{9}$ ). The residual interaction between those neutrons or protons not in closed shells or those with energies close to the Fermi energy is then approximated by the pairing-force Hamiltonian. The eigenstates of this Hamiltonian are known only for some very special systems ${ }^{10,11}$ ). In this paper, we reformulate the problem of determining the eigenstates of the pairing-force Hamiltonian and exhibit the explicit form of some of its eigenstates. Our eventual goal will be to apply our results to pairing-models of the nucleus; however, in this paper we formulate the problem in a very general form. (The details of applying our results to $\mathrm{Pb}^{206}, \mathrm{~Pb}^{204}$ and $\mathrm{Pb}^{202}$ are reported in the following paper). Although the general formulation may be well known, in the interests of readability and possible applications outside of nuclear physics, we discuss details which may be obvious to many.

Following the observation ${ }^{12}$ ) that there might exist correlations among the nucleons in a nucleus that are analogous to those of the electrons in a superconductor, many authors ${ }^{1-6}$ ) have applied the techniques developed for treating these correlations in superconductors to models of the nucleus. These authors use the methods of

$\uparrow$ Present address: Courant Institute of Mathematical Sciences, New York University, New York, New York.

${ }_{\uparrow}$ This work was supported by the National Science Foundation. 
the BCS theory of superconductivity ${ }^{13}$ ) or the Bogoliubov-Valatin canonical transformation ${ }^{14,15}$ ) to obtain approximate eigenstates of the model Hamiltonian, which is taken to be the pairing-force Hamiltonian. (We call the model that uses these approximate eigenstates of the pairing-force Hamiltonian the "superfluid model".) However, these approximate eigenstates are no longer eigenstates of the total number of particles and the approximations used to obtain them can only be justified for systems containing a large number of particles. Nevertheless the superfluid model has been used with considerable success to explain many of the observed properties of nuclei ${ }^{2-6}$ ).

In order to assess the accuracy of the superfluid model, some authors ${ }^{7,11,16}$ ) have diagonalized particular pairing-force Hamiltonians numerically and compared the exact results with those of the superfluid model. These computations are long and it is difficult to draw very general conclusions from them. In this paper we consider some eigenstates of the pairing-force Hamiltonian whose wave functions have simple analytical forms. These states can then be used to draw general conclusions about the exact eigenstates of the pairing-force Hamiltonian.

We begin our study of the exact eigenstates of the pairing-force Hamiltonian in sect. 2. This study takes advantage of the close similarity between the many-fermionpair system with pairing forces and the many-boson system with one-body forces that is obtained when each fermion pair of the many-fermion system is replaced by a single boson. We show that these two systems can be described by wave functions which differ only in that the many-fermion wave function is subject to subsidiary conditions which insure the fulfilment of the Pauli principle. For states whose wave functions satisfy the subsidiary conditions, the fermion-pair creation and annihilation operators can be formally treated as boson operators. We then show that the problem of determining the many-fermion wave function is equivalent to the problem of determining the eigenfunctions of a many-boson Schrödinger equation with an $N$-body interaction.

In sect. 3, we give the solutions of the Schrödinger equation for four classes of eigenstates: (1) the eigenstates of $N$ pairs in one multiply degenerate, single-particle level ${ }^{10}$ ). (2) the eigenstates of $N$ pairs in two multiply degenerate, single-particle levels, (3) the eigenstates of one pair in an arbitrary single-particle spectrum ${ }^{11}$ ), (4) a restricted class of $N$-pair eigenstates ${ }^{17}$ ). The eigenstates of the last class are natural generalizations of the one-pair states and the derivation of this class of solutions is the chief result of this paper. We shall see that many states of pairing models of physical systems belong to this class. (In particular, in the following paper, we shall show that the observed states of the isotopes of lead $\mathrm{Pb}^{206}, \mathrm{~Pb}^{204}$ and $\mathrm{Pb}^{202}$ belong to this class).

It should be noted that we only consider systems of identical fermions and that we do not consider systems with interactions between non-identical fermions such as neutrons and protons. 


\section{The Exact Eigenstates of the Pairing-Force Hamiltonian}

In writing the Hamiltonian of a many-fermion system interacting via pairing forces, we assume that the particles are contained in a fixed external potential well. We then let $(f \sigma)$ denote the single-particle quantum numbers and $\varepsilon_{f}$ the single-particle energy levels of this potential well, where $\sigma= \pm$ denotes states which are conjugate with respect to time reversal. The pairing-force Hamiltonian can then be written in second quantized form as

$$
H=\sum_{f} 2 \varepsilon_{f} \hat{N}_{f}-g \sum_{f}^{\prime} \sum_{f^{\prime}}^{\prime} b_{f}^{+} b_{f^{\prime}},
$$

where

$$
\begin{gathered}
\hat{N}_{f}=\frac{1}{2}\left(a_{f+}^{+} a_{f+}+a_{f-}^{+} a_{f-}\right), \\
b_{f}=a_{f-} a_{f+},
\end{gathered}
$$

and $a_{f \sigma}^{+}$and $a_{f \sigma}$ are fermion creation and annihilation operators which satisfy the anticommutation relations

$$
\left[a_{f \sigma}, a_{f^{\prime} \sigma^{\prime}}^{+}\right]_{+}=\delta_{\sigma \sigma^{\prime}} \delta_{f f^{\prime}}
$$

The primes on the last two sums in eq. (2.1) indicate that they are restricted to those values of $f$ contained in a set $S$ which is specified when the interaction is defined. The set $S$ is usually taken to comprise a convenient collection of levels near the Fermi energy of the system.

The derivation of a Schrödinger equation for the eigenstates of this Hamiltonian will be given in three parts. In the first part, we write $H$ as the sum of two operators, $H_{1}$ and $H_{2}$, which describe independent parts of the system. The eigenfunctions of $H$ are then products of an eigenfunction of $H_{1}$ with one of $H_{2}$. Since $H_{1}$ will be shown to represent the non-interacting particles of the system, its eigenstates are simple and the problem is reduced to a study of the eigenstates of $\mathrm{H}_{2}$. We start our consideration of the eigenstates of $\mathrm{H}_{2}$ in the second part of our derivation. Here we show that it is the Pauli principle and not the dynamics of the system which complicates the determination of these states. We then go on to formulate a method for treating the Pauli principle in which the matrix elements of $\mathrm{H}_{2}$ have the same formal appearance as those of the Hamiltonian of a many-boson system interacting with one-body forces. In the third part we obtain a Schrödinger equation for the eigenstates of $\boldsymbol{H}_{2}$ by requiring that the expectation value of $\mathrm{H}_{2}$ be stationary with respect to variations of the eigenstates. The resulting equation is formally the same as the Schrödinger equation for the eigenstates of a many-boson system with an $\mathrm{N}$-body interaction. Thus in our derivation of the eigenstates of $\mathrm{H}_{2}$, the complications of the Pauli principle are traded for the complications of an $\mathrm{N}$-body interaction in a many-boson system.

\subsection{THE SPLITTING OF $H$ INTO $H_{1}$ AND $H_{8}$}

We now split $H$ into two independent parts $H_{1}$ and $H_{2}$. The part $H_{1}$ will represent the non-interacting particles of the system while $\mathrm{H}_{2}$ will represent the remaining 
particles which interact with each other via pairing forces. This splitting is state dependent and will depend upon which levels in the set $S$ are occupied by unpaired particles.

The non-interacting particles of the system fall into two classes. The first class consists of those particles occupying single-particle states $(f \sigma)$ for which $f$ is not contained in the set $S$. These particles do not interact because the matrix elements of the interaction vanish for these values of $f$. The second class is made up of "unpaired" particles which occupy levels for which $f$ is contained in $S$. (An unpaired particle is defined as one which occupies a level $(f, \sigma)$ when the level $(f,-\sigma)$ is unoccupied.) These particles do not interact because the pairing force describes interactions between paired particles only.

The specification of the particles of this second class can be made explicit by introducing the operators ${ }^{16}$ )

$$
\hat{v}_{f}=a_{f+}^{+} a_{f+}-a_{f-}^{+} a_{f-},
$$

for $f$ belonging to the set $S$. Since $\left[\hat{v}_{f}, H\right]=0$, these operators represent constants of the motion and the eigenstates of $H$ are also eigenstates of the operators $\hat{v}_{f}$. The eigenvalues $v_{f}$ of the operators $\hat{v}_{f}$ can be obtained by considering the states of the non-interacting $(g=0)$ system for which they are $v_{f}=0, \sigma$. The physical interpretation of the eigenvalues $v_{f}=\sigma$ follows from the fact that for these values of $f$ the eigenstate of $H$ is also an eigenstate of the occupation number operators $a_{f \sigma}^{+} a_{f \sigma}$ and $a_{f-\sigma}^{+} a_{f-\sigma}$ with eigenvalues 1 and 0 , respectively. This is synonymous with the statement that those levels $(f \sigma)$ for which $v_{f}=\sigma$ are occupied by unpaired noninteracting particles. The eigenvalue $v_{f}=0$ corresponds to the two remaining possibilities for occupation of the pairs of levels $(f \pm)$, i.e., those pairs of levels $(f \pm)$ for which $v_{f}=0$ are either both occupied or both unoccupied. Thus an eigenstate of $H$ may be represented as a linear combination of states

$$
a_{f_{1} \sigma_{1}}^{+} \ldots a_{f_{M} \sigma_{M}}^{+} b_{f^{\prime} 1}^{+} \ldots b_{f^{\prime} N}^{+}|0\rangle
$$

where the $f_{i}$ are distinct and are fixed by the requirements $v_{f_{i}}=\sigma_{i}, i=1 \ldots M$, and the $f_{i}^{\prime}$ are allowed to range over the set of values for which $v_{f^{\prime}}=0, i=1 \ldots N$.

In accordance with current usage ${ }^{16}$ ) the operator

$$
\hat{v}=\sum_{f}^{\prime} \hat{v}_{f}^{2}
$$

will be called the seniority operator. It is a generalization of Racah's seniority operator ${ }^{18}$ ) now applicable to non-degenerate single-particle states. Each eigenvalue $v$ of $\hat{v}$ gives the number of unpaired particles which occupy levels contained in $S$.

Using the operators $\hat{v}_{f}$, we may now complete the splitting of $H$. Let

where

$$
H=H_{1}+H_{2},
$$

$$
H_{1}=\sum_{f} 2 \varepsilon_{f} \hat{N}_{f}
$$


where the sum is over the set $S_{1}$ of values of $f$, and

$$
H_{2}=\sum_{f} 2 \varepsilon_{f} \hat{N}_{f}-g \sum_{f} \sum_{f^{\prime}} b_{f}^{+} b_{f^{\prime}},
$$

where the sums are over the set $S_{2}$ of values of $f$. The set $S_{1}$ contains those levels which are not in $S$ plus those levels in $S$ for which $v_{f}=\sigma$. The set $S_{2}$ consists of those levels in $S$ for which $v_{f}=0$. Note that this splitting is state-dependent through its dependence on the set of quantum numbers $v_{f}$.

Since $H_{1}$ and $H_{2}$ commute and are constructed from independent sets of dynamical variables of the system, the eigenstates of $H$ are products of an eigenstate of $H_{1}$ with one of $\mathrm{H}_{2}$ and the eigenvalues of $\mathrm{H}$ are sums of the corresponding eigenvalues of $\mathrm{H}_{1}$ and $H_{2}$. The Hamiltonian $H_{1}$ represents a system of non-interacting particles in an external potential well. Its eigenstates are therefore

$$
a_{f_{1} \sigma_{1}}^{+} \ldots a_{f_{M} \sigma_{M}}^{+}|0\rangle
$$

and the corresponding energy eigenvalue is

$$
\varepsilon_{f_{1}}+\ldots+\varepsilon_{f_{M}}
$$

Thus, the problem of determining the eigenstates of $H$ has been reduced to the study of the eigenstates of $\mathrm{H}_{2}$.

Since the eigenstates of $\mathrm{H}_{2}$ only involve paired particles in the levels contained in $S_{2}$, a general expression for these states is

$$
|\psi\rangle=(N !)^{-\frac{1}{2}} \sum_{f_{1} \ldots f_{N}} \psi\left(f_{1} \ldots f_{N}\right) b_{f_{1}}^{+} \ldots b_{f_{N}}^{+}|0\rangle,
$$

where the factor $(N !)^{-\frac{1}{2}}$ is taken for convenience, and the sums on the $f_{i}$ are over those values of $f$ contained in $S_{2}$. In what follows we shall restrict all the quantum numbers $f$ to those values contained in $S_{2}$.

The wave function $\psi\left(f_{1} \ldots f_{N}\right)$ in eq. (2.6) is to be determined so that $|\psi\rangle$ is an eigenstate of $H_{2}$. It may be obtained by calculating the matrix elements of $H_{2}$ and diagonalizing the resultant matrix. The matrix elements of $H_{2}$ can be calculated using the commutation relations

$$
\begin{gathered}
{\left[b_{f}, \hat{N}_{f^{\prime}}\right]=\delta_{f f^{\prime}} b_{f},} \\
{\left[b_{f}, b_{f^{\prime}}^{+}\right]=\delta_{f f^{\prime}}\left(1-2 \hat{N}_{f}\right),}
\end{gathered}
$$

which result from the definitions (2.1a) and (2.1b) and the anticommutation relations (2.2). In general, however, this is a prohibitively long task and one must either use approximations (e.g., the superfluid model) or restrict the problem to some special cases for which $\psi\left(f_{1} \ldots f_{N}\right)$ has an especially simple form. It is the latter approach that we shall take in this paper. 
It is important to keep the state-dependence of $\mathrm{H}_{2}$ and its eigenstates (through the definition of the set $S_{2}$ ) in mind. This dependence is the so-called "blocking effect" which has been studied in the superfluid model by Soloviev ${ }^{4}$ ) and Wahlborn ${ }^{19}$ ). That is, those levels $f$ for which $v_{f}=\sigma$ are "blocked" and deleted from the set $S$ to leave the set of levels $S_{2}$ available to the interacting pairs of particles.

\subsection{THE TREATMENT OF THE PAULI PRINCIPLE}

We now show that the difficulties met in obtaining $\psi\left(f_{1} \ldots f_{N}\right)$ have their origin in the Pauli principle and not in the dynamics of the system. This is done by showing that $\psi\left(f_{1} \ldots f_{N}\right)$ may be readily obtained if we neglect the Pauli principle ${ }^{\dagger}$. Neglecting the Pauli principle would mean that the operators $b_{f}$ and $b_{f}^{+}$obey boson commutation relations. This simplifies the calculation of the matrix elements of operators and permits easy determination of $\psi\left(f_{1} \ldots f_{N}\right)$. After demonstrating these effects we go on to formulate a method in which the Pauli principle is satisfied but $b_{f}$ and $b_{f}^{+}$may nevertheless be treated formally as boson operators.

If we were to neglect the Pauli principle and replace every fermion pair by a single boson, then $\mathrm{H}_{2}$ would become

$$
H_{\mathrm{b}}=\sum_{f} 2 \varepsilon_{f} \beta_{f}^{+} \beta_{f}-g \sum_{f, f^{\prime}} \beta_{f}^{+} \beta_{f^{\prime}},
$$

where, instead of (2.7) and (2.8), we have

$$
\left[\beta_{f}, \beta_{f^{\prime}}^{+}\right]=\delta_{f f^{\prime}} .
$$

(The replacement of $\hat{N}_{f}$ by the boson number operator $\beta_{f}^{+} \beta_{f}$ follows from the interpretation of $\hat{N}_{f}$ as a number-of-fermion-pairs operator.) For simplicity here, we assume that $\varepsilon_{f} \neq \varepsilon_{f^{\prime}}$ for $f \neq f^{\prime}$. However, we do not impose this restriction on $\mathrm{H}_{2}$ when we consider its eigenstates.

Since $H_{\mathrm{b}}$ is a bilinear form in the boson operators, it may be diagonalized by a canonical transformation. It may be verified, by substituting into eq. (2.9), that the correct transformation is

$$
B_{\mathrm{p}}=g C_{\mathrm{p}} \sum_{f}\left(2 \varepsilon_{f}-E_{\mathrm{p}}\right)^{-1} \beta_{f},
$$

where $E_{\mathrm{p}}$ and $g C_{\mathrm{p}}$ are determined by

$$
\begin{gathered}
1=\sum_{f}\left(2 \varepsilon_{f}-E_{\mathrm{p}}\right)^{-1}, \\
\left(g C_{\mathrm{p}}\right)^{-2}=\sum_{f}\left(2 \varepsilon_{f}-E_{\mathrm{p}}\right)^{-2} .
\end{gathered}
$$

The $N$-boson eigenstates of $H_{\mathrm{b}}$ are then given by

$$
\left|\mathrm{p}_{1} \ldots \mathrm{p}_{N}\right\rangle=B_{\mathrm{p}_{1}}^{+} \ldots B_{\mathrm{p}_{N}}^{+}|0\rangle,
$$

+ This observation has already been made by Mrs. Høgaasen-Feldman ${ }^{11}$ ) in her study of the accuracy of the superfluid model. 
and the corresponding energy is

$$
E=E_{\mathrm{p}_{1}}+\ldots+E_{\mathrm{p}_{\mathrm{N}}} .
$$

If we rewrite (2.12) in a form similar to (2.6) the many-boson wave function $\psi_{\mathrm{b}}\left(f_{1} \ldots f_{N}\right)$ is given (up to a normalization constant) by

$$
\psi_{\mathrm{b}}\left(f_{1} \ldots f_{N}\right)=\sum_{P} P\left\{\prod_{k=1}^{N}\left(2 \varepsilon_{f_{k}}-E_{\mathrm{p}_{k}}\right)^{-1}\right\}
$$

where we have symmetrized the wave function

$$
\prod_{k=1}^{N}\left(2 \varepsilon_{f_{k}}-E_{p_{k}}\right)^{-1}
$$

which results from a direct substitution of (2.11) into (2.12), by summing over the $N$ ! permutations $P$ of the indices $\mathrm{p}_{1} \ldots \mathrm{p}_{N}$. We shall return to these results when we consider the eigenstates of $\mathrm{H}_{2}$.

We now formulate a method for treating the Pauli principle in which boson commutation rules may be used for the fermion-pair operators $b_{f}$ and $b_{f}^{+}$. In this method, the complications due to the Pauli principle are replaced by a set of subsidiary conditions on the wave function. In order to formulate this method, we return to the expansion (2.6) of the state $|\psi\rangle$. We first note that since $\psi\left(f_{1} \ldots f_{N}\right)$ is a many-fermion wave function, it should be a totally symmetric function of the fermion-pair variables $f_{1} \ldots f_{N}$. Also, since $b_{f}^{2}=0$, those values of $\psi\left(f_{1} \ldots f_{N}\right)$ with two or more arguments equal are arbitrary. We call these values of $\psi$ the "unphysical values". The physical values of $\psi\left(f_{1} \ldots f_{N}\right)$ are then those values for which $f_{1} \ldots f_{N}$ are all distinct. Since the unphysical values of $\psi$ are arbitrary, we are free to choose them in any convenient way. We shall show that a convenient choice for the unphysical values of $\psi$ is zero. We therefore require $\psi$ to satisfy

$$
\delta_{f_{i} f_{j}} \psi\left(f_{1} \ldots f_{N}\right)=0, \quad \text { all } i \neq j .
$$

We now demonstrate that the requirements (2.15) are a complete description of the Pauli principle and that for states whose wave functions satisfy eqs. (2.15) the $b_{f}$ may be formally treated as boson operators. That is, for states whose wave functions satisfy (2.15), we may neglect both the fact that $b_{f}^{2}=0$ and the presence of the term $2 \hat{N}_{f}$ in the commutation relations (2.8) for the $b_{f}$. First it is clear that $b_{f}^{2}=0$ is redundant for states whose wave functions satisfy (2.15). For (2.15) cancels precisely those terms which vanish in (2.6). Therefore we may neglect the fact that $b_{f}^{2}=0$. We now show that (2.15) also channels the contributions of $2 \hat{N}_{f}$ in the commutation relations of the $b_{f}$ by giving two examples.

As a first example of this cancellation, we consider the normalization of the state $|\psi\rangle$. Using (2.6), we have

$$
\langle\psi \mid \psi\rangle=(N !)^{-1} \sum_{\substack{f_{1} \\ f_{1}^{\prime} \ldots f_{N}}} \psi^{*}\left(f_{1}^{\prime} \ldots f_{N}^{\prime}\right) \psi\left(f_{1} \ldots f_{N}\right)\left\langle f_{1}^{\prime} \ldots f_{N}^{\prime} \mid f_{1} \ldots f_{N}\right\rangle,
$$


where we have written $\left|f_{1} \ldots f_{N}\right\rangle$ for the states $b_{f_{1}}^{+} \ldots b_{f_{N}}^{+}|0\rangle$. Using the commutation rules (2.7) and (2.8) for the $b$, we have

$$
\begin{aligned}
\left\langle f_{1}^{\prime} \ldots f_{N}^{\prime} \mid f_{1} \ldots f_{N}\right\rangle=\left\langle f_{2}^{\prime} \ldots f_{N}^{\prime}\left|b_{f^{\prime} 1} b_{f_{1}}^{+} b_{f_{2}}^{+} \ldots b_{f_{N}}^{+}\right| 0\right\rangle \\
=\left\langle f_{2}^{\prime} \ldots f_{N}^{\prime}\left|\left[b_{f_{1}}^{+} b_{f^{\prime} 1}+\delta_{f^{\prime} 1 f_{1}}\left(1-2 \hat{N}_{f_{1}}\right)\right] b_{f_{2}}^{+} \ldots b_{f_{N}}^{+}\right| 0\right\rangle \\
=\left\langle f_{2}^{\prime} \ldots f_{N}^{\prime}\left|\left\{b_{f_{1}}^{+} b_{f^{\prime} 1}+\delta_{f^{\prime} 1 f_{1}}\left[1-2\left(\delta_{f_{1} f_{2}}+\ldots+\delta_{f_{1} f_{N}}\right)\right]\right\}\right| f_{2} \ldots f_{N}\right\rangle .
\end{aligned}
$$

However, by (2.15), the term proportional to $\left(\delta_{f_{1} f_{2}}+\ldots+\delta_{f_{1} f_{N}}\right)$ does not contribute to (2.16). We therefore have

$$
\begin{aligned}
\left\langle f_{1}^{\prime} \ldots f_{N}^{\prime} \mid f_{1} \ldots f_{N}\right\rangle=\left\langle f_{2}^{\prime} \ldots f_{N}^{\prime}\left|\left[b_{f_{1}}^{+} b_{f^{\prime}{ }_{1}}+\delta_{f^{\prime} 1 f_{1}}\right]\right| f_{2} \ldots f_{N}\right\rangle \\
+\{\text { terms which do not contribute to }(2.16)\} .
\end{aligned}
$$

Continuing in this fashion we have

$$
\begin{aligned}
& \left\langle f_{1}^{\prime} \ldots f_{N}^{\prime} \mid f_{1} \ldots f_{N}\right\rangle=\sum_{P^{\prime}} P^{\prime}\left\{\delta_{f^{\prime} 1 f_{1}} \ldots \delta_{f^{\prime}{ }_{N} f_{N}}\right\} \\
& +\{\text { terms which do not contribute to }(2.16)\}
\end{aligned}
$$

where $\sum_{P^{\prime}} P^{\prime}$ is a sum over the $N !$ permutations $P^{\prime}$ of the indices $f_{1}^{\prime} \ldots f_{N}^{\prime}$. Substituting (2.17) into (2.16) we then obtain

$$
\langle\psi \mid \psi\rangle=\sum_{f_{1} \ldots f_{N}}\left|\psi\left(f_{1} \ldots f_{N}\right)\right|^{2}
$$

and the state $|\psi\rangle$ is normalized if

$$
\sum_{f_{1} \ldots f_{N}}\left|\psi\left(f_{1} \ldots f_{N}\right)\right|^{2}=1
$$

This is precisely the result we should have obtained had we used boson commutation relations for the $b_{f}$.

For our second example, we consider the expectation value of $H_{2}$. From eq. (2.5b), we have

$$
\left\langle\psi\left|H_{2}\right| \psi\right\rangle=\sum_{f} 2 \varepsilon_{f}\left\langle\psi\left|\hat{N}_{f}\right| \psi\right\rangle-g \sum_{f, f^{\prime}}\left\langle\psi\left|b_{f}^{+} b_{f^{\prime}}\right| \psi\right\rangle .
$$

In order to evaluate (2.19), we first use (2.6) to write

$$
\left\langle\psi\left|\hat{N}_{f}\right| \psi\right\rangle=(N !)^{-1} \sum_{\substack{f_{1}^{\prime} \ldots f_{N} \\ f^{\prime} 1 \ldots f^{\prime} N}} \psi^{*}\left(f_{1}^{\prime} \ldots f_{N}^{\prime}\right) \psi\left(f_{1} \ldots f_{N}\right)\left\langle f_{1}^{\prime} \ldots f_{N}^{\prime}\left|\hat{N}_{f}\right| f_{1} \ldots f_{N}\right\rangle
$$

and (2.7) to write

$$
\left\langle f_{1}^{\prime} \ldots f_{N}^{\prime}\left|\hat{N}_{f}\right| f_{1} \ldots f_{N}\right\rangle=\left(\delta_{f_{1} f}+\ldots+\delta_{f_{N} f}\right)\left\langle f_{1}^{\prime} \ldots f_{N}^{\prime} \mid f_{1} \ldots f_{N}\right\rangle .
$$

Using this result and (2.17), we have

$$
\left\langle\psi\left|\hat{N}_{f}\right| \psi\right\rangle=\sum_{i=1}^{N} \sum_{f_{1} \ldots f_{i-1} f_{i+1} \ldots f_{N}}\left|\psi\left(f_{1} \ldots f_{i-1} f f_{i+1} \ldots f_{N}\right)\right|^{2} .
$$


For the interaction term of (2.19) we need to evaluate

$$
\left\langle\psi\left|b_{f}^{+} b_{f^{\prime}}\right| \psi\right\rangle=(N !)^{-1} \sum_{\substack{f_{1} \ldots f_{N} \\ f^{\prime} 1 \ldots f^{\prime} N}} \psi^{*}\left(f_{1}^{\prime} \ldots f_{N}^{\prime}\right) \psi\left(f_{1} \ldots f_{N}\right)\left\langle f_{1}^{\prime} \ldots f_{N}^{\prime}\left|b_{f}^{+} b_{f^{\prime}}\right| f_{1} \ldots f_{N}\right\rangle
$$

Using (2.7), we have

$$
\begin{aligned}
\left\langle f_{1}^{\prime} \ldots f_{N}^{\prime}\left|b_{f}^{+} b_{f^{\prime}}\right| f_{1} \ldots f_{N}\right\rangle=\sum_{i=1}^{N} \delta_{f^{\prime} f_{i}}\left\langle f_{1}^{\prime} \ldots f_{N}^{\prime} \mid f_{1} \ldots f_{i-1} f f_{i+1} \ldots f_{N}\right\rangle \\
+\{\text { terms which do not contribute to (2.21) }\} .
\end{aligned}
$$

Using this result plus (2.17), we have

$$
\begin{aligned}
\left\langle\psi\left|b_{f}^{+} b_{f^{\prime}}\right| \psi\right\rangle=\sum_{i=1}^{N} \sum_{f_{1} \ldots f_{i-1} f_{i+1} \ldots f_{N}} \psi^{*}\left(f_{1} \ldots f_{i-1} f f_{i+1} \ldots f_{N}\right) & \\
& \times \psi\left(f_{1} \ldots f_{i-1} f^{\prime} f_{i+1} \ldots f_{N}\right) .
\end{aligned}
$$

Substituting (2.20) and (2.22) into (2.19), we have for the expectation value of $\mathrm{H}_{2}$

$$
\begin{aligned}
\left\langle\psi\left|H_{2}\right| \psi\right\rangle & =\sum_{f_{1} \ldots f_{N}}\left(2 \varepsilon_{f_{1}}+\ldots+2 \varepsilon_{f_{N}}\right)\left|\psi\left(f_{1} \ldots f_{N}\right)\right|^{2} \\
-g \sum_{i=1}^{N} & \sum_{f_{1} \ldots f_{i-1} f_{i+1} \ldots f_{N} f f^{\prime}} \psi^{*}\left(f_{1} \ldots f_{i-1} f f_{i+1} \ldots f_{N}\right) \psi\left(f_{1} \ldots f_{i-1} f^{\prime} f_{i+1} \ldots f_{N}\right) .
\end{aligned}
$$

Again, the same result would have been obtained if we had used boson commutation rules for the $b_{f}$.

Continuing in this fashion, it can be readily verified that the matrix elements of any operator can be calculated using boson commutation relations for the $b_{f}$ when the wave function satisfies (2.15). Thus the effects of the Pauli principle are completely described by the set of requirements (2.15) on the wave function.

\subsection{THE SCHRÖDINGER EQUATION FOR $\psi\left(f_{1} \ldots f_{N}\right)$}

We now obtain a Schrödinger equation for $\psi\left(f_{1} \ldots f_{N}\right)$ from the variational principle ${ }^{20}$ )

$$
\frac{\delta\left\langle\psi\left|H_{2}-E\right| \psi\right\rangle}{\delta \psi^{*}\left(f_{1} \ldots f_{N}\right)}=\frac{\delta\left\langle\psi\left|H_{2}-E\right| \psi\right\rangle}{\delta \psi\left(f_{1} \ldots f_{N}\right)}=0 .
$$

We require $\psi\left(f_{1} \ldots f_{N}\right)$ to satisfy $(2.15)$ so that we may use boson commutation relations for the $b_{f}$ and we require the state $|\psi\rangle$ to be normalized so that $\psi\left(f_{1} \ldots f_{N}\right)$ satisfies (2.18).

In order to restrict $\psi$ to those functions which satisfy (2.15), we let

$$
\psi\left(f_{1} \ldots f_{N}\right)=\theta\left(f_{1} \ldots f_{N}\right) \varphi\left(f_{1} \ldots f_{N}\right),
$$

where $\varphi\left(f_{1} \ldots f_{N}\right)$ is a symmetric function of its arguments and $\theta\left(f_{1} \ldots f_{N}\right)$ is defined by

$$
\theta\left(f_{1} \ldots f_{N}\right)= \begin{cases}1, & \text { if } f_{1} \ldots f_{N} \text { are all distinct } \\ 0, & \text { if any } f_{i}=f_{j}\end{cases}
$$


A general expression for $\theta$ is

$$
\theta\left(f_{1} \ldots f_{N}\right)=\prod_{i<j}^{N}\left(1-\delta_{f_{i} f_{j}}\right) .
$$

Notice that the unphysical values of $\varphi$ are arbitrary since the unphysical values of $\theta$ vanish.

For the $\psi$ given by (2.25), we rewrite the variational principle (2.24) as

$$
\frac{\delta\left\langle\psi\left|H_{2}-E\right| \psi\right\rangle}{\delta \varphi^{*}\left(f_{1} \ldots f_{N}\right)}=\frac{\delta\left\langle\psi\left|H_{2}-E\right| \psi\right\rangle}{\delta \varphi\left(f_{1} \ldots f_{N}\right)}=0 .
$$

Using (2.23) and (2.25), we have

$$
\begin{aligned}
\frac{\delta\left\langle\psi\left|H_{2}-E\right| \psi\right\rangle}{\delta \varphi^{*}\left(f_{1} \ldots f_{N}\right)} & =\theta\left(f_{1} \ldots f_{N}\right)\left[\left(2 \varepsilon_{f_{1}}+\ldots+2 \varepsilon_{f_{N}}-E\right) \varphi\left(f_{1} \ldots f_{N}\right)\right. \\
& \left.-g \sum_{i=1}^{N} \sum_{f} \theta\left(f_{1} \ldots f_{i-1} f f_{i+1} \ldots f_{N}\right) \varphi\left(f_{1} \ldots f_{i-1} f f_{i+1} \ldots f_{N}\right)\right] .
\end{aligned}
$$

Using the relation

$$
\theta\left(f_{1} \ldots f_{N}\right) \theta\left(f_{1} \ldots f_{i-1} f f_{i+1} \ldots f_{N}\right)=\theta\left(f_{1} \ldots f_{N}\right)\left[1-\sum_{j \neq i}^{N} \delta_{f_{j} f}\right]
$$

(which follows from the definition (2.26) of $\theta$ ) and (2.29), we obtain the Schrödinger equation

$$
\begin{aligned}
\theta\left(f_{1} \ldots f_{N}\right)\left[\left(2 \varepsilon_{f_{1}}+\ldots+\right.\right. & \left.2 \varepsilon_{f_{N}}-E\right) \varphi\left(f_{1} \ldots f_{N}\right) \\
& \left.\quad-g \sum_{i=1}^{N} \sum_{f}\left(1-\sum_{j \neq i}^{N} \delta_{f_{j} f}\right) \varphi\left(f_{1} \ldots f_{i-1} f f_{i+1} \ldots f_{N}\right)\right]=0
\end{aligned}
$$

for $\varphi$. Since the second equation (2.28) gives the same equation for $\varphi^{*}$, we may require $\varphi$ to be real. The factor $\theta$ in (2.30) indicates that it is identically satisfied for the unphysical values of $\varphi$. A natural way to define these values of $\varphi$ is to ignore the factor $\theta$ in (2.30) and require $\varphi$ to satisfy

$$
\begin{aligned}
\left(2 \varepsilon_{f_{1}}+\ldots+2 \varepsilon_{f_{N}}-E\right) \varphi & \left(f_{1} \ldots f_{N}\right) \\
& -g \sum_{i=1}^{N} \sum_{f}\left(1-\sum_{j \neq i}^{N} \delta_{f_{j} f}\right) \varphi\left(f_{1} \ldots f_{i-1} f f_{i+1} \ldots f_{N}\right)=0
\end{aligned}
$$

for all values of $f_{1} \ldots f_{N}$. Notice that if $f_{1} \ldots f_{N}$ are distinct then only $\varphi$ with $f_{1} \ldots f_{i-1} f f_{i+1} \ldots f_{N}$ distinct occur in the second term of (2.31). This suggests two interpretations of (2.31). One can assume that only the physical values of $\varphi$ are determined by (2.31) and that the unphysical values are undefined or one can assume that all values of $\varphi$ are determined by (2.31). We shall use both interpretations when we consider some explicit solutions of (2.31) in sect. 3 .

Eq. (2.31) has the formal appearance of a Schrödinger equation for the eigenstates of an $N$-boson system with an $N$-body interaction. Its real, symmetric solutions 
characterize the eigenstates $|\psi\rangle$ of $H_{2}$ in the $\left|f_{1} \ldots f_{N}\right\rangle$ representation. These states are normalized when $\varphi$ satisfies

$$
\sum_{f_{1} \ldots f_{N}} \theta\left(f_{1} \ldots f_{N}\right) \varphi^{2}\left(f_{1} \ldots f_{N}\right)=1 .
$$

In the next section we shall consider some explicit solutions of eq. (2.31).

\section{The Eigenstates of the Pairing-Force Hamiltonian for Four Types of Systems}

We now consider four types of systems for which we can write the explicit form of $\varphi$ which satisfies eq. (2.31). The types are characterized by the number of pairs $N$, the levels $\varepsilon_{f}$ contained in $S$ and the pairing-interaction strength $g$. The specification of the fourth type requires in addition certain restrictions placed on the particular state of the system under consideration.

The systems for which $S$ contains one and two multiply-degenerate levels make up our first two types. The eigenstates of the second type of system are generalizations of the well-known ${ }^{10}$ ) eigenstates of the first type. The one-pair systems characterize the third type. These well-known ${ }^{11}$ ) eigenstates are treated in some detail since they serve as an introduction to the fourth type ${ }^{17}$ ) which we call "a restricted class of $N$-pair eigenstates". The eigenstates of this class will be shown to be natural generalizations of the one-pair states. The precise nature of the restrictions will be specified in subsect. 3.4.

For the first two types of systems we assume that only the physical components of $\varphi$ are determined by (2.31) and we leave the unphysical components undefined. There are no unphysical components of $\varphi$ for the one-pair systems of the third type. And finally, for the fourth type we assume that both the physical and the unphysical components of $\varphi$ are determined by (2.31).

\section{1. $N$ PAIRS IN ONE MULTIPLY-DEGENERATE LEVEL}

The systems for which $S$ contains one multiply-degenerate level are sometimes called the "strong coupling limit" of the pairing interaction ${ }^{21}$ ). Let $\varepsilon$ be the energy and $\Omega$ the pair degeneracy of the single level in $S$. (The pair degeneracy is defined as the number of different values of $f$ contained in $S$.) Eq. (2.31) for the physical values of $\varphi$ for the ground state (i.e., the seniority $v=0$ state) of $N$ pairs in this system is

$$
(2 N \varepsilon-E) \varphi\left(f_{1} \ldots f_{N}\right)-g \sum_{i=1}^{N} \sum_{S}^{\prime} \varphi\left(f_{1} \ldots f_{i-1} f f_{i+1} \ldots f_{N}\right)=0,
$$

where $\sum_{f}$ is a sum over those values of $f$ in $S$ which are not equal to $f_{1} \ldots f_{i-1}$ $f_{i+1} \ldots f_{N}$ and is therefore over $(\Omega-N+1)$ values of $f$. The solution of (3.1) is $\varphi=$ constant, and the corresponding eigenvalue is

$$
E_{0}=2 N \varepsilon-g N(\Omega-N+1) \text {. }
$$


The first excited states are the seniority $v=2$ states and they are the result of breaking up one pair in the ground state. Therefore, the energy of these states is the energy of $(N-1)$ pairs in a single degenerate level of energy $\varepsilon$ and pair degeneracy $\Omega-2$ (since $S_{2}$ is formed by deleting from $S$ the two levels occupied by the two unpaired particles) plus the energy of the two unpaired particles, i.e.,

$$
E_{1}=2 N \varepsilon-g(N-1)(\Omega-N) .
$$

Continuing in this fashion, the energy of the $n$th excited states, which are the seniority $v=2 n$ states, is

$$
E_{n}=2 N \varepsilon-g(N-n)(\Omega-N-n+1) ; \quad n=0 \ldots N .
$$

The value of $\varphi$ for the $n$th excited states can be derived from the normalization condition and is

$$
\varphi_{n}=\left[\frac{(\Omega-N-n) !}{(\Omega-2 n) !}\right]^{\frac{1}{2}}
$$

Since these results have been treated by Mottelson ${ }^{10}$ ), we do not consider them in any more detail here.

\section{2. $N$ PAIRS IN TWO MULTIPLY-DEGENERATE LEVELS}

When $S$ contains two multiply-degenerate levels, the eigenvalue problem can be reduced to the solution of a simple algebraic equation and explicit expressions can be given for the wave function. To achieve this reduction we first write the quantum number $f$ as

$$
f=(n, m) ; \quad n=0.1 ; \quad m=1 \ldots \Omega_{n},
$$

where $\Omega_{n}$ is the pair degeneracy of the level whose energy is $\varepsilon_{n}$. For convenience, we shift the energy scale so that $\varepsilon_{0}=0$ and $\varepsilon_{1}=\varepsilon$. Eq. (2.31) for the physical values of $\varphi$ for the seniority $v=0$ states of $N$ pairs in the system is

$$
\begin{aligned}
{\left[2 \sum_{i=1}^{N} n_{i} \varepsilon-E\right] } & \left(n_{1} m_{1} \ldots n_{N} m_{N}\right) \\
& -g \sum_{i=1}^{N} \sum_{n, m}^{\prime} \varphi\left(n_{1} m_{1} \ldots n_{i-1} m_{i-1} n m n_{i+1} m_{i+1} \ldots n_{N} m_{N}\right)=0,
\end{aligned}
$$

where $\sum_{n, m}^{\prime}$ is a sum over those values of $(n, m)$ in $S$ which do not equal $n_{1} m_{1} \ldots n_{i-1} m_{i-1} n_{i+1} m_{i+1} \ldots n_{N} m_{N}$. We assert that

$$
\varphi\left(n_{1} m_{1} \ldots n_{N} m_{N}\right)=\varphi\left(n_{1} \ldots n_{N}\right)
$$

and note that any symmetric function of $N$ variables $n_{i}\left(n_{i}=0,1\right)$ is a function of the single variable ${ }^{\dagger} \mu$, where

$$
\mu=\sum_{i=1}^{N} n_{i}
$$

$\dagger$ We are indebted to the paper of Mattis and Lieb ${ }^{22}$ ) for this observation. 
Therefore,

$$
\varphi\left(n_{1} m_{1} \ldots n_{N} m_{N}\right)=\varphi(\mu) .
$$

Note that $\mu$ gives the number of pairs in the configuration $n_{1} m_{1} \ldots n_{N} m_{N}$ which occupy the level $n=1$. Using (3.5), (3.3) becomes

$$
\left[\omega_{\mu}-E\right] \varphi(\mu)-A_{\mu} \varphi(\mu+1)-B_{\mu} \varphi(\mu-1)=0,
$$

where

$$
\begin{gathered}
\omega_{\mu}=2 \mu \varepsilon-g(N-\mu)\left(\Omega_{0}-N+\mu+1\right)-g \mu\left(\Omega_{1}-\mu+1\right), \\
A_{\mu}=g(N-\mu)\left(\Omega_{1}-\mu\right), \\
B_{\mu}=g \mu\left(\Omega_{0}-N+\mu\right) .
\end{gathered}
$$

Comparing (3.6a) with (3.2), we see that $\omega_{\mu}$ is the sum of the ground state energies of $(N-\mu)$ pairs in the level $n=0$ and $\mu$ pairs in the level $n=1$.

Eq. (3.6) can be solved by standard techniques ${ }^{23}$ ). The energy $E$ is a root of

$$
\omega_{0}-E=\frac{A_{0} B_{1}}{\omega_{1}-E-\frac{A_{1} B_{2}}{\omega_{2}-E-\quad \ldots}}
$$

and the wave function is given by

$$
\begin{array}{r}
\varphi(\mu)=\frac{B_{\mu} \varphi(\mu-1)}{A_{\mu} B_{\mu+1}} \\
\cdots \\
\quad-\frac{A_{N-1} B_{N}}{\omega_{N}-E}
\end{array}
$$

for $\mu \geqq 1$. The quantity $\varphi(0)$ is determined by the normalization. The $(N+1)$ roots of (3.7) correspond to the $(N+1)$ seniority $v=0$ states of the system. The seniority $v=2$ states can be obtained from the same eqs. (3.6)-(3.8) by reducing $N$ by one and blocking (subsect. 2.1) the two levels occupied by the two unpaired particles. In this way all of the states of the system can be obtained.

\subsection{THE ONE-PAIR SYSTEMS}

For $N=1$ and arbitrary $S$, eq. (2.31) is

$$
\left(2 \varepsilon_{f_{1}}-E\right) \varphi\left(f_{1}\right)-g \sum_{f} \varphi(f)=0 .
$$

The normalized solutions are

$$
\varphi(f)=g C_{\mathrm{p}}\left(2 \varepsilon_{f}-E_{\mathrm{p}}\right)^{-1},
$$


where $E_{\mathrm{p}}$ is a root of

$$
\begin{gathered}
1=g \sum_{f}\left(2 \varepsilon_{f}-E_{\mathrm{p}}\right)^{-1}, \\
\left(g C_{\mathrm{p}}\right)^{-2}=\sum_{f}\left(2 \varepsilon_{f}-E_{\mathrm{p}}\right)^{-2} .
\end{gathered}
$$

Note that these states are the same as the one-boson states (2.12) of the Hamiltonian (2.9). This is due to the absence of Pauli-principle complications in the one-pair systems.

Since the solution of equations similar to (3.11) will play an important role in the work that follows, we discuss its solution in some detail here. We first separate from the quantum numbers $f$ the quantum number $n$, which indexes the different energy levels of the potential well. Then

$$
\varepsilon_{f}=\varepsilon_{n} \text {. }
$$

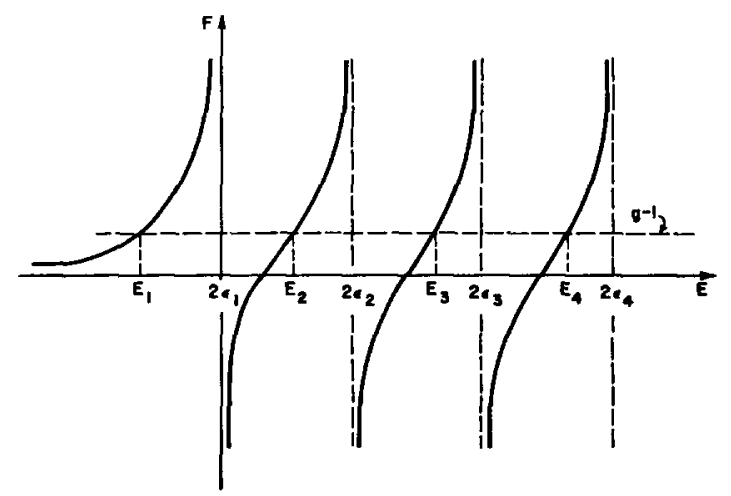

Fig. 1. The qualitative behaviour of $F(E)$ and the graphical solution of eq. (3.13).

We also define $\Omega_{n}$ as the pair degeneracy of the level $n$, i.e., $\Omega_{n}$ is the number of values of $f$ for which $\varepsilon_{f}=\varepsilon_{n}$. And finally, we define $F(E)$ by

$$
F(E)=\sum_{n} \Omega_{n}\left(2 \varepsilon_{n}-E\right)^{-1}
$$

Eq. (3.11) can then be written as

$$
F\left(E_{\mathrm{p}}\right)=g^{-1} \text {. }
$$

In fig. 1, we give a qualitative picture of $F(E)$ and show how the roots of (3.13) can be obtained graphically. The roots $E_{\mathrm{p}}$ of (3.13) are labelled so that

$$
\lim _{g \rightarrow 0^{+}} E_{\mathrm{p}}=2 \varepsilon_{\mathrm{p}}
$$

The qualitative behaviour of $F(E)$ and the roots of (3.13) should be kept in mind when we treat the next class of eigenstates which are natural generalizations of these one-pair states. 


\subsection{A RESTRICTED CLASS OF N-PAIR EIGENSTATES}

We now consider a restricted class of $N$-pair eigenstates which are natural generalizations of the preceding one-pair states. That is, the wave functions of these states have the form of a symmetrized product of $N$ one-pair wave functions and the energy has the form of a sum of $N$ pair-energies in complete analogy with the many-boson states (2.13) and (2.19). These one-pair wave functions and energies are obtained from one-pair Schrödinger equations in which the pairing-interaction strength $g$ has been replaced by an effective pairing-interaction strength $g_{i}$ which in turn depends upon the $N$ pair-energies. We shall see that the $N$-pair wave functions in the form described above must be restricted by the requirement that no two pair-energies be equal, in order that the wave functions satisfy the Schrödinger equation.

Let us write eq. (2.31) for both the physical and unphysical components of $\varphi$ as

$$
\begin{aligned}
\left(2 \varepsilon_{f_{1}}+\ldots+2 \varepsilon_{f_{N}}-E\right) & \varphi\left(f_{1} \ldots f_{N}\right)-g \sum_{i=1}^{N} \sum_{f} \varphi\left(f_{1} \ldots f_{i-1} f f_{i+1} \ldots f_{N}\right) \\
& +g \sum_{i \neq j}^{N} \varphi\left(f_{1} \ldots f_{i-1} f_{j} f_{i+1} \ldots f_{j-1} f_{j} f_{j+1} \ldots f_{N}\right)=0 .
\end{aligned}
$$

We assume that $\varphi$ and $E$ have the same form as (2.14) and (2.13), i.e.,

$$
\begin{gathered}
\varphi\left(f_{1} \ldots f_{N}\right)=\sum_{P} P\left\{\prod_{k=1}^{N}\left(2 \varepsilon_{f_{k}}-E_{\mathrm{p}_{k}}\right)^{-1}\right\}, \\
E=E_{\mathrm{p}_{1}}+\ldots+E_{\mathrm{p}_{N}},
\end{gathered}
$$

where the pair-energies $E_{\mathrm{p}_{1}}$ are to be determined so that (3.16) is a solution of (3.15) and $\sum_{P} P$ is a sum over the $N$ ! permutation $P$ of the indices $p_{1} \ldots p_{N}$. The only restriction we impose upon (3.16) is the requirement that the $E_{\mathrm{p}_{t}}$ be distinct, i.e.,

$$
E_{\mathrm{p}_{i}} \neq E_{\mathrm{p}_{j}}, \quad \text { for } i \neq j .
$$

We now substitute (3.16) and (3.17) into (3.15) and consider the three terms of (3.15) separately.

Using (3.16) and (3.17), the first two terms of (3.15) are

$$
\begin{gathered}
\left(2 \varepsilon_{f_{1}}+\ldots+2 \varepsilon_{f_{N}}-E\right) \varphi\left(f_{1} \ldots f_{N}\right)=\sum_{i=1}^{N} \sum_{P} P\left\{\prod_{k \neq i}^{N}\left(2 \varepsilon_{f_{k}}-E_{\mathrm{p}_{k}}\right)^{-1}\right\} \\
-g \sum_{i=1}^{N} \sum_{f} \varphi\left(f_{1} \ldots f_{i-1} f f_{i+1} \ldots f_{N}\right) \\
=-g \sum_{i=1}^{N} \sum_{P} P\left\{\left[\sum_{\mathcal{P}}\left(2 \varepsilon_{f}-E_{\mathrm{p} i}\right)^{-1}\right] \prod_{k \neq i}^{N}\left(2 \varepsilon_{f_{k}}-E_{\mathrm{p}_{\mathrm{k}}}\right)^{-1}\right\} .
\end{gathered}
$$

In order to evaluate the third term of (3.15), we consider

$$
\begin{aligned}
\varphi\left(f_{1} \ldots f_{i-1} f_{j} f_{i+1} \ldots\right. & \left.f_{j-1} f_{j} f_{j+1} \ldots f_{N}\right) \\
& =\sum_{P} P\left\{\left(2 \varepsilon_{f_{j}}-E_{\mathrm{p}_{i}}\right)^{-1}\left(2 \varepsilon_{f_{j}}-E_{\mathrm{p}_{j}}\right)^{-1} \prod_{k \neq i, j}^{N}\left(2 \varepsilon_{f_{k}}-E_{\mathrm{p}_{k}}\right)^{-1}\right\} .
\end{aligned}
$$


The assumption (3.18) allows us to use the partial fraction expansion

$$
\begin{aligned}
\left(2 \varepsilon_{f_{j}}-E_{\mathrm{p}_{i}}\right)^{-1}\left(2 \varepsilon_{f_{j}}-E_{\mathrm{p}_{j}}\right)^{-1}=\left(E_{\mathrm{p}_{j}}-E_{\mathrm{p}_{i}}\right)^{-1} & {\left[\left(2 \varepsilon_{f_{j}}-E_{\mathrm{p}_{j}}\right)^{-1}-\left(2 \varepsilon_{f_{j}}-E_{\mathrm{p}_{i}}\right)^{-1}\right] } \\
& =\left(1+P_{i j}\right)\left(E_{\mathrm{p}_{j}}-E_{\mathrm{p}_{i}}\right)^{-1}\left(2 \varepsilon_{f_{j}}-E_{\mathrm{p}_{j}}\right)^{-1},
\end{aligned}
$$

where $P_{i j}$ is the transposition $p_{i} \rightleftarrows p_{j}$. Using (3.21), we have

$$
\begin{aligned}
\varphi\left(f_{1} \ldots f_{i-1} f_{j} f_{i+1} \ldots f_{j-1}\right. & \left.f_{j} f_{j+1} \ldots f_{N}\right) \\
& =\sum_{P} P\left\{\left(1+P_{i j}\right)\left(E_{\mathrm{p}_{j}}-E_{\mathrm{p}_{i}}\right)^{-1} \prod_{k \neq i}^{N}\left(2 \varepsilon_{f_{k}}-E_{\mathrm{p}_{k}}\right)^{-1}\right\} \\
& =2 \sum_{P} P\left\{\left(E_{\mathrm{p}_{j}}-E_{\mathrm{p}_{i}}\right)^{-1} \prod_{k \neq i}^{N}\left(2 \varepsilon_{f_{k}}-E_{\mathrm{p}_{k}}\right)^{-1}\right\},
\end{aligned}
$$

where we have used the re-arrangement theorem for permutation groups

$$
\left(\sum_{P} P\right) P_{i j}=\sum_{P} P \text {. }
$$

Inserting (3.19), (3.20) and (3.22) into (3.15), we obtain

$$
\sum_{i=1}^{N} \sum_{P} P\left\{\left[1+2 g \sum_{j \neq i}^{N}\left(E_{\mathbf{p}_{j}}-E_{\mathbf{p}_{i}}\right)^{-1}-g \sum_{f}\left(2 \varepsilon_{f}-E_{\mathbf{p}_{i}}\right)^{-1}\right] \prod_{k \neq i}^{N}\left(2 \varepsilon_{f_{k}}-E_{\mathbf{p}_{k}}\right)^{-1}\right\}=0,
$$

which gives the coupled system of equations

$$
1+2 g \sum_{j \neq i}^{N}\left(E_{\mathrm{p}_{j}}-E_{\mathrm{p}_{i}}\right)^{-1}-g \sum_{f}\left(2 \varepsilon_{f}-E_{\mathrm{p}_{i}}\right)^{-1}=0 ; \quad i=1 \ldots N,
$$

for the pair-energies $E_{\mathrm{p}_{i}}$. These equations may be written in a form similar to (3.13) if we define

$$
g_{i}=g\left[1+2 g \sum_{j \neq i}^{N}\left(E_{\mathrm{p}_{j}}-E_{\mathrm{p}_{i}}\right)^{-1}\right]^{-1}
$$

as the "effective pairing-interaction strength for the $i$ th pair". Eqs. (3.24) then become

$$
F\left(E_{\mathrm{p}_{i}}\right)=g_{i}^{-1} ; \quad i=1 \ldots N,
$$

which are completely analogous to' (3.13).

The restrictions on this class of eigenstates are expressed in the requirement that eqs. (3.26) have roots $E_{\mathrm{p} i}$ which are distinct. For without these restrictions, the partial fraction expansion (3.21) would not be possible and (3.16) would not be a solution of (3.15). Whether these restrictions are satisfied or not depends very strongly upon the system and state being considered and must be carefully checked when this solution of the Schrödinger equation is used. Sometimes the restrictions may be discussed without actually solving (3.26) for the $E_{\mathbf{p}_{i}}$. For example, consider those states for which

$$
\lim _{g \rightarrow 0^{+}} E_{\mathrm{p}_{i}} \neq \lim _{g \rightarrow 0^{+}} E_{\mathrm{p}_{j}}, \quad \text { for } i \neq j
$$


For these states the restrictions are satisfied for $g=0$ and, since the roots of (3.26) are continuous functions of $g$ at $g=0$, they are satisfied in a region about $g=0$. This example includes all the states of systems whose single-particle spectra are doubly degenerate such as the Nilsson model of the nucleus ${ }^{9}$ ).

The domain of validity of eqs. (3.26) can be extended by allowing the $\mathrm{E}_{\mathrm{p}_{t}}$ to be complex. When complex $E_{\mathrm{p}_{i}}$ occur as roots of (3.26) they occur in complex conjugate pairs. This preserves the reality of the energy $E$, which is the sum of the $E_{\mathrm{p}_{1}}$, and the wave function $\varphi$. (When the $E_{\mathrm{p}_{i}}$ occur in complex conjugate pairs the operation of taking the complex conjugate of $\varphi$ is equivalent to that permutation of the $E_{p_{i}}$ which interchanges each complex $E_{\mathrm{p}_{i}}$ with its complex conjugate. However, by the rearrangement theorem (3.23), this leaves $\varphi$ unchanged.) The occurrence of complex $E_{\mathrm{p}_{i}}$ in complex conjugate pairs is also consistent with eqs. (3.26). For example, if $E_{\mathrm{p}_{1}}=E_{\mathrm{p}_{2}}^{*}$ then, by (3.25), $g_{1}=g_{2}^{*}$ and, since $F^{*}(E)=F\left(E^{*}\right)$, this implies that $E_{\mathrm{p}_{1}}=E_{\mathrm{p}_{2}}^{*}$. However, the existence and interpretation of complex roots of (3.26) depend upon the details of the system and state being treated and cannot be discussed in general. Examples do occur in the applications to the isotopes of lead and they will be described in the following paper.

For states that do satisfy these restrictions, the effect of the Pauli principle can be absorbed in the set of effective interaction strengths $g_{i}$. The solution of (3.26) is then greatly facilitated by the presence of the one function $F(E)$ evaluated for different values of its argument in each of the equations. Once this function is tabulated, eqs. (3.26) can be solved by iteration.

It is important to note that (3.16) is a many-body wave function of a particularly simple form. It is not a symmetrized product of one-pair functions. Although it has the form of a symmetrized product of one-pair functions, the products in the sum are not separately solutions of the Schrödinger equation (3.15). This is evident from the use of the re-arrangement theorem (3.23) in the derivation of (3.26). For, if any permutation or group of permutations is deleted from $\sum_{P} P$, then (3.16) would no longer satisfy (3.15).

To summarize these results, we have the normalized wave functions

$$
\varphi\left(\hat{1} \ldots f_{N}\right)=C \sum_{P} P\left\{\prod_{k=1}^{N}\left(2 \varepsilon_{f_{k}}-E_{\mathrm{p}_{k}}\right)^{-1}\right\},
$$

and the corresponding energy is

$$
E=E_{\mathrm{p}_{1}}+\ldots+E_{\mathrm{p}_{N}}
$$

where the $E_{\mathbf{p}_{i}}$ are $N$ distinct roots of

$$
F\left(E_{\mathrm{p} i}\right)=g_{i}^{-1}
$$

and where $F$ and $g_{i}$ are defined by (3.12) and (3.25), respectively. The normalization constant $C$ is determined by (2.32). In the following paper, we shall apply these results to pairing models of some even isotopes of lead. 


\section{References}

1) S. T. Belyaev, Mat. Fys. Medd. Dan. Vid. Selsk. 31, No. 11 (1959)

2) L. S. Kisslinger and R. A. Sorensen, Mat. Fys. Medd. Dan. Vid. Selsk. 32, No. 9 (1960)

3) S. G. Nilsson and O. Priov, Mat. Fys. Medd. Dan. Vid. Selsk. 32, No. 16 (1961)

4) V. G. Soloviev, Thesis, Joint Institute for Nuclear Research, Dubna, U.S.S.R. (1961)

5) J. J. Griffin and M. Rich, Phys. Rev. Lett. 3 (1959) 342

6) C. J. Gallagher, Jr. and V. G. Soloviev, Mat. Fys. Skr. Dan. Vid. Selsk. 2, No. 2 (1962)

7) A. K. Kerman, R. D. Lawson and M. H. MacFarlane, Phys. Rev. 124 (1961) 162

8) J. P. Elliot and A. M. Lane, Handbuch der Physik, vol. 39 (Springer-Verlag, Berlin, 1957) p. 241

9) S. G. Nilsson, Mat. Fys. Medd. Dan. Vid. Selsk. 29, No 16 (1955)

10) B. R. Mottelson, in The many-body problem, Grenoble Université, Ecole d'Ete de Physique Théorique Les Houches (John Wiley and Sons, New York, 1959)

11) J. Hogaasen-Feldman, Nuclear Physics 28 (1961) 258

12) A. Bohr, B. R. Mottelson and D. Pines, Phys. Rev. 110 (1958) 936

13) T. Bardeen, L. N. Cooper and J. R. Schrieffer, Phys. Rev. 108 (1957) 1175

14) N. N. Bogoliubov, Nuovo Cim. 7 (1958) 794

15) J. G. Valatin, Nuovo Cim. 7 (1958) 843

16) A. Pawlikowski and V. Rybarska, JETP (Soviet Physics) 16 (1963) 388

17) R. W. Richardson, Phys. Lett. 3 (1963) 277

18) G. Racah, Rev. 62 (1942) 438

19) S. Wahlborn, Nuclear Physics 37 (1962) 554

20) J. D. Landau and E. M. Lifshitz, Quantum mechanics (Addison-Wesley Publ. Co., Reading, Mass., 1958) chapt. 3, p. 55 